\title{
La révolution face aux « victimes du pouvoir arbitraire » : l'abolition des lettres de cachet et ses conséquences
}

The Revolution and the "victims of arbitrary power" : the abolition of the lettres de cachet and its consequences (1789-1792)

Jeanne-Marie Jandeaux

\section{(2) OpenEdition \\ Journals}

\section{Édition électronique}

URL : https://journals.openedition.org/ahrf/12293

DOI : 10.4000/ahrf.12293

ISSN : $1952-403 X$

\section{Éditeur :}

Armand Colin, Société des études robespierristes

\section{Édition imprimée}

Date de publication : 1 juin 2012

Pagination : 33-60

ISBN : 978-2-7489-0161-0

ISSN : 0003-4436

\section{Référence électronique}

Jeanne-Marie Jandeaux, « La révolution face aux « victimes du pouvoir arbitraire » : l'abolition des lettres de cachet et ses conséquences ", Annales historiques de la Révolution française [En ligne], 368| avril-juin 2012, mis en ligne le 01 juin 2015, consulté le 22 avril 2022. URL : http:// journals.openedition.org/ahrf/12293 ; DOI : https://doi.org/10.4000/ahrf.12293 


\title{
LA RÉVOLUTION FACE AUX «VICTIMES DU POUVOIR ARBITRAIRE " : L'ABOLITION DES LETTRES DE CACHET ET SES CONSÉQUENCES
}

\author{
Jeanne-Marie JANDEAUX
}

\begin{abstract}
Les lettres de cachet, érigées dès 1789 en symbole honni de l'Ancien Régime, ont connu une lente agonie, marquée par les hésitations et le doute. Alors même que cette incarnation de la monarchie absolue et de l'arbitraire royal faisait apparemment l'unanimité contre elle, les députés de l'Assemblée nationale constituante ont attendu mars 1790 avant d'enfin en prononcer l'abolition. La crainte des conséquences d'une telle mesure retient en effet les députés de l'Assemblée qui nomment en novembre 1789 un Comité des lettres de cachet chargé de préparer l'action du législateur et d'enquêter sur cette masse obscure, inquiétante et hétérogène de prisonniers du roi et correctionnaires, encore détenue dans des maisons de force en vertu de lettres de cachet. Les débats puis le texte du décret final traduisent la prudence de députés partagés entre le désir de rendre à la lumière ces « victimes de l'oppression » et la peur du désordre qui pourrait s'ensuivre.
\end{abstract}

Mots-clés : lettres de cachet, arbitraire, Justice retenue, Comité des lettres de cachet, Révolution, prisons.

En 1789, les lettres de cachet, dont la légende noire commence à s'écrire, symbolisent déjà les excès et les insuffisances de l'Ancien Régime dans son entier. Les enjeux de leur abolition dépassent largement la simple expression d'un ordre royal direct ayant pour principal objet l'enfermement de sujets parce qu'ils s'opposent soit à la volonté et à la loi du roi, soit à la volonté et à la loi de leur famille, dans un subtil parallèle entre ordre social et ordre familial, respect de l'État et respect du père. Non seulement le pouvoir souverain et la clairvoyance du roi, source de 
toute justice, sont mis en cause ainsi que rétrospectivement tous les ordres qu'il a pu délivrer, mais en outre le système de gouvernement de l'Ancien Régime se trouve mis à mal par la suppression de cet instrument d'ordre administratif, politique et judiciaire, utilisé à grande échelle à partir du $\mathrm{XVIII}^{\mathrm{e}}$ siècle $^{1}$. La question de l'abolition des lettres de cachet, devenues au fur et à mesure du siècle avant tout un outil de régulation des conflits familiaux par la monarchie, place la justice retenue du roi, et donc par conséquent l'essence même du pouvoir royal, au cœur des débats.

La tâche du Comité des lettres de cachet, institué le 24 novembre 1789, s'annonce extrêmement complexe, étant donné le nombre important de détenus et la difficulté, voire l'impossibilité effective de connaître les motifs réels de leur enfermement. Le dépouillement des archives du Comité des lettres de cachet conservées aux Archives nationales (soussérie $\mathrm{D} / \mathrm{V})^{2}$, constituées dans leur grande majorité d'états de détenus par ordre du roi et de la correspondance reçue autour de certains dossiers, donne la mesure de la mission confiée au Comité : d'abord légiférer en vue de sceller le destin des « ordres arbitraires », ensuite veiller à l'application du décret d'abolition voté les 13 et 16 mars 1790 par l'Assemblée et sanctionné par lettres patentes du roi le 26 mars.

Concrètement, une question cruciale se pose dès l'été 1789 : quel sort réserver aux milliers de prisonniers par lettre de cachet qui croupissent encore dans prisons d'État, couvents, monastères, ou hôpitaux généraux à travers tout le royaume? Pour les hommes de la Révolution, il s'agit là d'un véritable dilemme. La tentation première d'ouvrir les portes des maisons de force fait long feu car on sait qu'à défaut d'avoir été jugés, les prisonniers du roi ou prisonniers de famille ne sont pas pour autant innocents et sont même souvent incarcérés pour de très bonnes raisons. Comment donc parvenir à concilier le rejet de «l'arbitraire » et du « despotisme » avec la sûreté du royaume et la tranquillité des familles? Cette hésitation permanente tenaille le législateur puis l'exécutif en charge de l'application du décret d'abolition, sous la pression constante des prison-

(1) Jacques-Guy Petit estime à environ 200000 les ordres envoyés entre 1660 et 1780. Jacques-Guy PETIT (dir.), Histoire des galères, bagnes et prisons, XIII ${ }^{e}-X X^{e}$ siècles : introduction à l'histoire pénale de la France, Toulouse, Privat, 1991, p. 68. Au sujet de la multiplication et de la démocratisation des lettres de cachet « pour affaires de famille » au XVIII siècle, voir l'analyse marquante d'Arlette Farge et de Michel Foucault sur le cas parisien. Arlette Farge, Michel Foucault, Le Désordre des familles, Paris, Gallimard, 1982.

(2) Pierre-Dominique Cheynet, Archives nationales (Paris). Série D (missions des représentants du peuple et comités des assemblées). Répertoire numérique, Paris, Archives nationales, 2007, p. 50-52. 
niers, de leurs gardiens et des familles qui font entendre avec force leur voix dans les débats.

\section{Les hommes de la Révolution face à la délicate question des lettres de cachet}

\section{La création du Comité des lettres de cachet dans un contexte troublé et confus}

L'Assemblée nationale constituante néglige pendant de longs mois la question des lettres de cachet, institution pourtant déjà fortement discréditée dans la dernière décennie de l'Ancien Régime par des abus récurrents, les attaques des parlementaires et des philosophes ou encore les récits édifiants de fameux prisonniers du $\mathrm{roi}^{3}$. Le monarque lui-même formule le vœu de leur abolition dans la Déclaration des intentions du roi lue devant l'Assemblée le 23 juin 1789 où il invite les États généraux

« à chercher et à lui proposer les moyens les plus convenables de concilier l'abolition des ordres connus sous le nom de lettres de cachet, avec le maintien de la sûreté publique et avec les précautions nécessaires, soit pour ménager dans certains cas l'honneur des familles, soit pour réprimer avec célérité les commencements de la sédition, soit pour garantir l'État des effets d'une intelligence criminelle avec les puissances étrangères $»^{4}$.

Nombreux sont les cahiers de doléances qui abordent le sujet, privilégiant la réforme à une abolition pure et simple. Beaucoup fustigent le secret de la procédure et insistent sur le fait que l'accusé doit connaître les faits qui lui sont reprochés afin de se défendre et éventuellement de se pourvoir contre les demandeurs ${ }^{5}$. D'autres vont jusqu'à demander réparation pour les victimes et la poursuite des ministres et des exécuteurs de l'ordre arbitraire ${ }^{6}$ mais on se prononce généralement pour la conservation des lettres de cachet de famille dont l'utilité est reconnue. Certains cahiers s'accordent également à justifier l'existence des lettres de cachet

(3) Mirabeau, Des lettres de cachet et des prisons d'État, s.1., 1782; Simon-Nicolas-Henri Linguet, Mémoires sur la Bastille et sur la détention de M. Linguet, Londres, T. Spilsbury, 1783 ; Henri Masers de LAtude, Le despotisme dévoilé ou mémoires de Henri Masers de Latude, détenu pendant trente-cinq ans dans diverses prisons d'État, Paris, Lejay, 1790, notamment.

(4) Article 15.

(5) Cahiers du tiers état de Bar-sur-Seine.

(6) Cahiers du clergé de Beauvais. 
mais comme seul expédient pour s'assurer de la personne d'un accusé avant de le remettre entre les mains des « juges naturels ».

Le premier pas vers l'abolition est franchi avec l'adoption de la Déclaration des Droits de l'Homme et du Citoyen ${ }^{7}$ dont les articles 7, 8 et 9 auraient été votés «par réaction contre la pratique des lettres de cachet ${ }^{8}$. Leurs trois rédacteurs, un magistrat, Duport, et deux avocats, Mounier et Target, y établissent le principe de stricte légalité des délits et des peines. L'emprisonnement relève désormais uniquement de la loi pénale 9 . Les dispositions pénales de la Déclaration s'érigent ainsi comme des remparts contre l'arbitraire de l'Ancien Régime et en particulier contre ces enfermements sans décision de justice et selon le « bon plaisir du roi », qui le symbolisaient. C'est au cours des débats sur l'adoption d'une « Déclaration des droits des hommes » en préambule de la Constitution qu'ont été évoquées le $1^{\text {er }}$ août 1789 , pour la première fois, les lettres de cachet. Il faut toutefois attendre le 9 octobre 1789 et la lecture devant l'Assemblée de la lettre d'un religieux incarcéré pour que les députés envisagent l'abolition des lettres de cachet et la création d'un comité susceptible de régler la délicate question des prisonniers du roi ${ }^{10}$, suivant en cela les préconisations de certains cahiers de doléances ${ }^{11}$. S'éloignant d'une logique manichéiste simpliste qui aurait voulu que la Justice triomphe des erreurs de l'Ancien Régime en brisant les chaînes de toutes les innocentes victimes de l'arbitraire, l'Assemblée constituante, reprenant la proposition du Comte de Dortan ${ }^{12}$, institue le 23 octobre 1789 un comité censé clarifier la situation et préparer son œuvre législative.

L'œuvre immense des comités créés pour fonder un nouvel ordre politique, judiciaire et social en France débute à l'automne 1790; l'élection des membres du Comité des lettres de cachet le 24 novembre 1789 intervient dans un climat d'urgence et d'extrême tension : un vent de révolte et de sédition commence à souffler sur les lieux de détention

(7) Votée le 2 octobre 1789.

(8) Jean-Marie CARbasse, « Le droit pénal dans la Déclaration des droits » DroIT, revue française de théorie juridique, vol. 8, Paris, 1988, p. 123-134.

(9) Roberto Martucci, « En attendant Pelletier de Saint-Fargeau », AHRF, n 328, 2002, p. $77-104$.

(10) Proposition du Comte de Montmorency. Voir Philippe NÉgRIn, La réforme de la lettre de cachet au XVIII siècle, Paris, E. Larose, 1906, p. 94 sq.

(11) Par exemple la noblesse de Bordeaux suggère la création d'un comité au sein des États généraux qui aurait pour mission de dresser une liste des prisonniers et de statuer sur leur cas. Philippe NÉGRIN, La réforme de la lettre de cachet, op. cit., p. 93.

(12) Émile Laurent, Jérôme Mavidal (dir.), Archives parlementaires de 1787 à 1860, t. IX, Paris, P. Dupont, 1875-1889, p. 483. 
du royaume. Au cour des paradoxes nés dans ces jours de trouble et d'intense effervescence, on constate à la fois un appel à la remise en liberté de prisonniers détenus dans les geôles de l'Ancien Régime, peu relayé dans les actes au final, et des vagues d'arrestation frappant, sans intervention aucune de la justice, fauteurs de trouble et prétendus contrerévolutionnaires à Paris comme dans tout le royaume ${ }^{13}$. Dans ce contexte, la question du sort à réserver aux prisonniers par ordre du roi s'avère brûlante. Le bruit des événements révolutionnaires s'est vite répandu dans les prisons et fait naître beaucoup d'espoir. Ce qui se produit dans le fort des îles Sainte-Marguerite ${ }^{14}$ en décembre 1789 est particulièrement révélateur. Les détenus par lettre de cachet

" attendoient dans une inaction respectueuse que l'Assemblée nationalle brisant jusques à la dernière verge du despotisme ministériel réalisat en leur faveur ce principe protecteur qui, prescrivant avec une sévérité inexorable tout ordre arbitraire, établit que nul homme ne peut être accusé, arrêtté ni détenu, que dans les cas déterminés par la loy selon les formes qu'elle a prescrittes $\rangle^{15}$.

Après s'être aperçus toutefois le 22 décembre

" que les soldats invalides et ceux de la compagnie franche de Castelanne [sic] préposés à la garde du fort ne leur opposeroient point de résistance s'ils vouloient se resaisir de leur liberté [...], [ils] s'étoient présentés chés le commandant de la place et lui avoient nottifié qu'ils cessoient dès ce moment de se regarder comme ses prisonniers et qu'ils alloient se rendre dans une terre libre ».

Le 25 décembre les cinq prisonniers arrivent dans la rade de la Croisette ${ }^{16}$ et se dirigent vers Grasse pour se placer « sous la protection de la nation, du roy et de la loy ». Les consuls grassois prient alors les députés de l'Assemblée constituante de leur accorder la liberté et d'appliquer de cette façon en leur faveur un des grands principes de l'« immortelle déclaration des droits » :

« Daignés donc achever de briser leurs liens; rendés-les à la patrie

(13) Roberto MartuccI, art. cit., p. 93-94.

(14) Fort de l'Île Sainte-Marguerite, Îles de Lérins.

(15) AN D/V/3. Souligné dans le texte.

(16) On mentionne que deux prisonniers sont restés au fort, l'un par crainte des suites que pourrait avoir sa fuite, l'autre à cause de son grand âge. 
qu'ils peuvent servir encore, à un roy qu'ils adorent, à l'humanité qui les réclame, sauvés-les à jamais de ce pouvoir terrible et immoral qui faisoit qu'au lieu de trouver des citoyens dans l'empire, on n'y auroit bientôt rencontré que des sujets. Ne souffrés pas en un mot qu'après avoir reconquis la liberté pour tous les François, il y en ait encore qui portent les fers du despotisme $[\ldots] »$

À la fin de l'année 1789, pour les hommes ayant adopté les idées nouvelles, du simple fait de leur statut, de la procédure et des principes au nom desquels ils ont été mis au secret, et tout simplement du symbole qu'ils représentent, les prisonniers par ordre du roi sont par définition innocents et leur remise en liberté est l'un des premiers devoirs de la Nation.

«L'évasion » des détenus du fort des îles Sainte-Marguerite fait grand bruit et, le 6 janvier 1790, trois prisonniers du Château de Ferrières s'appuient sur cet exemple pour réclamer au « président des États généraux » leur $"$ amnistie $»{ }^{17}$. Dans le même temps, le commandant du Château d'If assure les députés qu' « en brisant les liens des malheureuses victimes du despotisme », ils donnent « une nouvelle existance [sic] à l'âme sensible d'un ancien militaire qui depuis dix sept ans qu'il a l'honneur de commander au château d'Iff [sic], gémit du sort des infortunés que des ordres tiranniques [sic] y détiennent ${ }^{18}$.

Devant le danger d'une libération générale et sans discernement, le maire de Paris, Bailly, tente d'interpeller l'Assemblée nationale sur l'urgence d'entamer une réflexion préalable à l'abolition des lettres de cachet afin d'en prévenir les conséquences qui pourraient s'avérer dramatiques :

«L'heureuse révolution qui rend la Nation libre nous affranchit pour jamais de ces actes du despotisme ministériel qui, sans forme, sans instruction, privoient les citoyens de leur liberté. Il n'existera plus de lettres de cachet et par une conséquence nécessaire, on doit voir tomber les fers des infortunés qui ne les portent que par un abus de pouvoir. Mais le moment présent où ces malheureux pourroient bénir la main qui les délivre est encore malheureusement un instant de trouble, peut-être de sédition [...]. Ne seroit-il pas dangereux dans ce moment de rendre sans réflexion à la cité des hommes qui en ont été arrachés sans légalité il est

(17) $\mathrm{AN} \mathrm{D} / \mathrm{V} / 2$.

(18) Ibid., D/V/3. 
vrai, mais presque toujours avec de justes motifs $»^{19}$ ?

Bailly pointe avec acuité le dilemme devant lequel se trouvent les députés confrontés à l'existence de ces prisonniers détenus en vertu d' « ordres illégaux » sans avoir subi le joug de la loi et la sentence de la justice : «Que doit-on faire par apport [sic] à eux : les retenir irrévocablement seroit une injustice, les faire sortir à l'instant seroit peut-être une imprudence $»^{20} \ldots$

\section{Les débats à l'Assemblée et les décrets des 15 janvier et 16 mars 1790.}

Les débats à l'Assemblée constituante autour de la question des lettres de cachet traduisent les hésitations des députés partagés entre la volonté d'afficher aux yeux de tous la proclamation d'un nouvel ordre basé sur l'affirmation de la légalité des peines et la défense de la liberté individuelle, et des réticences certaines à libérer massivement, dans un climat tendu et troublé, des milliers d'individus potentiellement dangereux, mis dans les fers « presque toujours avec de justes motifs » (Bailly). On est loin d'une condamnation unanime du régime des lettres de cachet lorsque, le 12 octobre 1789, le député Deschamps émet des réserves quant à l'idée d'une libération massive et sans précaution et ne manque pas de demander «si c'est par amour pour l'humanité qu'on voudrait rejeter dans la société ceux que le repos et le salut de la société a exigé d'en soustraire $\gg^{21} \ldots$ Les députés élus au Comité des lettres de cachet, Fréteau de Saint-Just, Mirabeau, Boniface de Castellane et Barère de Vieuzac, nommé président ${ }^{22}$, sont chargés de la lourde mission de préparer l'œuvre du législateur en la matière, soit, avant tout, dresser un état le plus exhaustif possible des détenus par lettres de cachet et des motifs de leur enfermement. Le Comité est également fortement impliqué dans la réorganisation des prisons. Cette mission échoue de prime abord à son membre le plus emblématique, le Comte de Mirabeau détenu à maintes reprises en vertu de lettres de cachet ${ }^{23}$. Dans les archives du Comité, aucun document ne témoigne de son action, à l'exception d'un modeste billet et de quelques lettres qui lui sont adressées. Il serait cependant l'auteur

(19) Ibid.

(20) Ibid.

(21) Archives parlementaires, t. IX, p. 412-413.

(22) Ibid., t. X, p. 249.

(23) Guy Chaussinand-Nogaret, Mirabeau, Paris, Le Seuil, 1982. 
d'un rapport sur le régime des prisons et les " maisons d'amélioration », probablement rédigé au début de l'année 1790 mais jamais officiellement déposé au Comité ${ }^{24}$. Deux lettres de Barère en font mention, la première en date du 21 septembre 1790, comportant cette remarque sèche : « Nous verrions au reste, avec beaucoup de plaisir que vous voulussiez bien partager les travaux du Comité, l'éclairer de vos lumières et faire au plutôt votre rapport sur les maisons de correction $»^{25}$ et qui reçoit une réponse toute aussi sèche de Mirabeau, la seconde en date du 24 novembre 1790 soulignant que « personne ne peut mieux traiter une pareille matière que l'auteur de l'excellent ouvrage des lettres de cachet $»^{26}$. À en croire leur omniprésence dans les archives, c'est à Boniface de Castellane et Barère de Vieuzac que semble revenue la part majeure du travail effectué. En août 1791, un seul secrétaire-commis est employé pour les seconder; rédigeant et enregistrant toute la correspondance reçue et expédiée, il dresse aussi à partir des états de prisonniers reçus, les tables alphabétiques de toutes les personnes détenues dans le royaume et de toutes les maisons et prisons dont elles sont issues, avec renvoi en marge aux pièces les concernant ${ }^{27}$.

Le Comité des lettres de cachet débute ses travaux à la fin de l'année 1789. Le 2 janvier 1790, Boniface de Castellane, reprenant des observations envoyées par le Comte de Saint-Priest ${ }^{28}$, annonce à l'Assemblée que celui-ci n'a pas été en mesure de donner les renseignements demandés et «que le ministre ne connaît même pas la plupart des noms des détenus ». Il propose alors un décret, aussitôt adopté par l'Assemblée le 15 janvier suivant, stipulant que les personnes ayant sous leur surveillance des prisonniers par ordre du roi ont pour obligation d'envoyer des états contenant les noms de ces derniers, les motifs et dates de la détention et « l'extrait des ordres en vertu desquels ils ont été emprisonnés ». L'objectif est de « vider successivement toutes les prisons illégales, en prenant cependant des précautions nécessaires pour ne pas compro-

(24) « Rapport sur les maisons d'amélioration au nom du Comité des lettres de cachet». Voir Henri De Bégouen, «Un rapport inédit de Mirabeau sur le régime des prisons » Revue d'économie politique, 1887, p. 491-512. Renée MARTINAGE, « Les origines de la pénologie dans le code pénal de 1791 » dans Michel Vovelle (dir.), La Révolution et l'ordre juridique privé, rationalité ou scandale? Actes du colloque d'Orléans, 11-13 septembre 1986, Orléans, PUF, p. 15-29.

(25) $\mathrm{AN} \mathrm{D} / \mathrm{V} / 6$.

(26) Ibid.

(27) Ibid., registre de correspondance du Comité des lettres de cachet, Paris, le 8 août 1791.

(28) François-Emmanuel Guignard de Saint-Priest, alors en charge de la Maison du Roi. 
mettre la sûreté publique $»^{29}$.

Par mesure de prudence, l'abolition est donc repoussée. Le 7 février, le député Voidel, de Sarreguemines, intervient devant la Chambre en vue « d'empêcher un parricide » et plaide en faveur du sieur Rousseau, officier de la petite chancellerie du Parlement de Nancy, dont le fils de 24 ans s'est engagé cinquante et une fois et a tiré sur un garde ${ }^{30}$. Ce dernier étant sur le point de sortir de prison, le député demande « que M. le Président soit autorisé à écrire au procureur général, pour que ce fils trop coupable soit retenu dans les prisons jusqu'à ce que la famille elle-même en sollicite la liberté ou consente à ce qu'elle lui soit accordée ». À cette sollicitation explicite de lettre de cachet, Le Chapelier et Mirabeau opposent le fait que seuls les juges peuvent condamner et évoquent déjà la création des tribunaux de famille. La lecture du Rapport au nom du Comité des lettres de cachet du Comte de Castellane devant les députés le 20 février marque une étape essentielle vers l'abolition. Son contenu nourri par les événements et les débats des derniers mois traduit une lente maturation de la réflexion du Comité quant au destin des milliers de prisonniers suspendus à l'adoption d'un décret. Boniface de Castellane s'excuserait presque de la prudence dont font preuve les membres du comité :

«Messieurs, c'est avec une grande répugnance que nous nous sommes vus forcés de retarder si longtemps à vous proposer de rendre la liberté aux victimes du pouvoir arbitraire qui gémissent encore dans les fers; mais telles étaient les funestes conséquences du despotisme ministériel, qu'une partie des maux qu'ils avaient produits devait se faire sentir dans les premiers jours de la liberté. Les innocents et les coupables, ceux qui ont conservé l'usage de la raison, et ceux qui l'ont perdue, se trouvant confondus ensemble dans les lieux de douleur que vous allez détruire; la sureté que vous devez à la nation entière vous a fait une loi d'apporter quelques précautions à l'entière suppression des prisons illégales. Vous avez remarqué que, parmi ceux qu'elles renfermaient, quelques-uns étaient déjà condamnés, que d'autres étaient prévenus de crimes, et vous avez reconnu l'impuissance où vous étiez de vous livrer à l'instant même aux sentiments d'humanité qui vous pressaient de ne point retarder un jour à faire jouir ceux qui avaient le plus souffert de l'ancien ordre des choses de tous les droits dont la constitution nouvelle doit leur assurer

(29) Archives Parlementaires, t. XI, p. 66.

(30) Ibid., t. XI, p. 488-489. 
l'exercice $[\ldots] »^{31}$

Comment distinguer les innocents des coupables? Comment libérer sans attendre les premiers tout en protégeant les citoyens des seconds? Boniface de Castellane distingue quatre classes de prisonniers « illégalement détenus », d'abord " ceux juridiquement accusés d'aucun crime ", ensuite les aliénés, enfin les prisonniers condamnés en justice et enfermés par commutation de peine, et ceux simplement décrétés de prise de corps. Le député s'attarde sur les spécificités de chacune de ces catégories de prisonniers : pour les premiers, principalement des «correctionnaires » ou prisonniers de famille, la liberté semble certes s'imposer; pourtant le Comité préconise un délai pour ménager les familles et le Comte, à mot couvert, exprime le désir, partagé par une majorité, de ne pas élargir sans précaution des individus potentiellement dangereux et sources de scandale. Les troisième et quatrième catégories sont bien celles qui posent de véritables cas de conscience, car ayant été soustraits d'une condamnation en justice pour tomber sous le coup d'une lettre de cachet, les individus concernés sont susceptibles de subir une double peine si l'on choisit de les remettre entre les mains des juges. Le projet de décret d'abolition des lettres de cachet et d'élargissement des détenus est âprement débattu article par article les semaines suivantes, le sort des prisonniers « juridiquement accusés d'aucun crime » déchaînant les passions et les craintes. L'abbé Maury estime que le projet de décret qui prévoit leur remise en liberté sans restriction, « accorde la liberté à des hommes coupables peutêtre des plus grands forfaits $»$ :

«S'il existe des innocents dans les prisons d'État, n'y-a-t-il pas aussi un bien plus grand nombre de personnes accusées sans preuves? À la vérité, ce sont des maris qui ont empoisonné leurs femmes, des fils qui ont empoisonné leurs pères. Il n'existe aucune accusation légale contre ces personnes : les rendrez-vous pour cela à la société $»^{32}$ ?

L'article premier est finalement adopté dans la soirée du 13 mars, intégrant toutes les réserves exprimées pendant les débats au sujet des "prisonniers de famille $»^{33}: 1$ 'Assemblée ordonne que tous les prisonniers par lettre de cachet soient remis en liberté dans les six semaines suivant la publication du décret, à l'exception des individus condamnés, décrétés

(31) Ibid., t. XI, p. 661.

(32) Ibid., t. XII, p. 160-161.

(33) Ibid., t. XII, p. 160-162. 
de prise de corps, des aliénés ou encore - amendement au projet initial des personnes enfermées pour des motifs « très graves » à la sollicitation de leur famille. On admet de fait, pour ces derniers, que la justice retenue ait agi comme palliatif de la justice déléguée, faute de preuves ou en vue d'éviter le scandale généré par un procès et une condamnation. Les quinze articles suivants ne sont discutés et adoptés que le 16 mars $^{34}$ : au cour du décret, l'article 10 prononce enfin l'abolition des lettres de cachet; les autres articles s'attachent à spécifier le traitement réservé aux différents types de détenus : les personnes décrétées ou faisant l'objet d'une plainte en justice seront jugées, mais leur peine ne pourra excéder quinze ans (articles $3,4,5)^{35}$, celles condamnées légalement poursuivent leur détention qui ne peut excéder dix ans (art. 7) ${ }^{36}$. Les prétendus aliénés doivent recevoir la visite de médecins et être interrogés par des juges dans les trois mois suivant la publication du décret, avant qu'on ne statue sur leur sort (art. 9). Quant aux prisonniers de famille, leur remise en liberté générale, signifiée par l'article 1, est modérée par l'article 6 stipulant que l'élargissement est soumis au fait qu'aucune plainte n'ait été déposée en justice contre eux dans les trois mois.

Le Comité a préparé également l'après détention : l'article 12 prévoit des expédients pour permettre aux prisonniers de rentrer dans leur famille ou dans leur communauté religieuse et l'article 13 précise que les officiers municipaux doivent s'assurer que les plus démunis puissent obtenir du travail dans des ateliers de charité. L'information des anciens prisonniers et la mise à jour des coupables sont enfin rendues possibles par l'obligation faite aux ministres de communiquer les mémoires et instructions sur lesquels furent délivrées les lettres de cachet (article 11) ${ }^{37}$. Enfin le Comité se donne les moyens de veiller à l'application du présent décret (art. 14, 15, 16) en réclamant dans les trois mois à venir aux commandants, directeurs et supérieurs, un état des personnes élargies, interrogées, visitées, renvoyées devant les tribunaux ou encore détenues

(34) Ibid., t. XII, p. 201-203.

(35) Excepté dans les cas d'assassinat, de poison ou d'incendie, où la détention à perpétuité pourra être prononcée.

(36) Dans les deux cas, cette durée maximale de détention inclut les années déjà purgées.

(37) Cet article suscite beaucoup de réserve chez les ministres. Dans une lettre adressée à Barère au sujet de la demande de communication des pièces de son dossier par le sieur Dagonneau de Bussy, Guignard observe « qu'il y auroit peut-être de l'inconvénient à donner trop de latitude à l'exécution de l'article 11 » et qu' « on ne peut se dissimuler que la disposition qu'il renferme est déjà rigoureuse par son application à des ordres déjà anciens, qu'elle peut donner lieu à de fâcheuses recherches et à des divisions dans les familles $[\ldots] »$. AN D/V/4. 
dans leurs maisons, état déposé aux archives du district avec dépôt de doubles aux archives du département et à l'Assemblée nationale. Tous les détenteurs de prisonniers par ordre arbitraire sont jugés responsables de l'application du décret sous l'œil vigilant des juges, des directoires des départements, des districts et des municipalités (article 16).

Le 16 mars 1790, après des mois d'attente, l'Assemblée nationale abolit finalement les lettres de cachet devenues le symbole des erreurs et des insuffisances de l'Ancien Régime, d'un mode de gouvernement et d'un système judiciaire à présent dénoncés et rejetés; beaucoup plus qu'à l'abolition elle-même, c'est à ses conséquences que s'est attaché le Comité des lettres de cachet, à savoir la destinée de milliers de prisonniers coupables ou innocents, dangereux ou inoffensifs, sur laquelle il convient de statuer au cas par cas. Hors de question désormais de céder à la folle tentation qu'ont eue certains d'ouvrir les portes des maisons de force de l'Ancien Régime au nom de la défense des droits de l'homme et de la lutte contre l'oppression. La rédaction puis l'adoption du décret se sont faites dans la douleur et son exécution, étant donné l'hétérogénéité des détenus concernés et les subtilités du texte, s'annonce extrêmement délicate.

\section{Le cri des « victimes du pouvoir arbitraire »}

Pendant les longs mois que durent les débats et la préparation du décret d'abolition par le Comité des lettres de cachet, les prisonniers par ordre du roi n'en peuvent plus d'attendre que l'immense espoir né des premiers pas de la Révolution se concrétise. La rumeur des événements s'est propagée jusque dans leurs geôles et le discours révolutionnaire fait d'eux des victimes. Toutefois, même si Robespierre ${ }^{38}$ et à son instar la grande majorité des députés clament que la Révolution n'aura pas achevé son œuvre tant qu'il y aura encore des opprimés, la peur retient les députés. Peur avant tout de la masse d'individus concernés et de l'opacité qui les entoure. Combien sont-ils? Qui les détient et dans quelles conditions? Peur ensuite du désordre social et familial que pourrait générer la remise en liberté d'individus séquestrés pour des motifs de dérangement et d'inconduite, malgré la vigueur des plaidoyers en leur faveur. Les correctionnaires et autres prisonniers du roi sont donc pris 
dans cette profonde ambivalence qui fait d'eux à la fois des coupables et des victimes et plonge dans l'incertitude l'opinion et les députés de la Nation.

\section{Combien y a-t-il de prisonniers par lettre de cachet et comment se répartissent-ils dans le royaume fin 1789 - début 1790 ?}

Il est difficile d'estimer précisément le nombre de détenus par ordre du roi dans le royaume à la fin de l'Ancien Régime. Claude Quétel avance le chiffre de 7000 à $8000^{39}$. La très grande majorité d'entre eux $(80 \%)$ sont incarcérés à la demande de leur famille. Les dossiers de lettres de cachet conservés dans les fonds des intendances en Province et aux Archives de la Bastille pour la lieutenance générale de Paris permettent certes d'évaluer le nombre de lettres de cachet expédiées sur une période donnée mais en aucun cas d'établir le nombre d'embastillés par ordre du roi à une époque précise ${ }^{40}$. Cette donnée échappe à l'administration royale qui abandonne le plus souvent, faute de moyens, de temps et d'information, le suivi des prisonniers à leur famille et à leurs geôliers.

Sous l'appellation commune de « maisons de force» se cache au $\mathrm{XVIII}^{\mathrm{e}}$ siècle une grande diversité de lieux de détention recevant des prisonniers par ordre du roi; forts et prisons d'État n'en accueillent qu'une minoritét ${ }^{41}$, la majorité se trouvant concentrée dans des hôpitaux généraux et surtout des communautés religieuses, dans une logique de répartition des individus par sexe et par rang social. Dans le cas des lettres de cachet de famille, le choix repose sur les moyens financiers de la famille et l'effort consenti pour son correctionnaire. Un vaste réseau de cinq à six cents maisons de force s'est ainsi développé dans tout le royaume ${ }^{42}$, en marge des prisons traditionnelles et au gré du formidable accroissement des lettres de cachet de famille au cours du siècle.

Deux établissements, l'hôpital de Bicêtre et l'hôpital de la Salpê-

(39) Claude Qú́TeL, De par le Roy, essai sur les lettres de cachet, Toulouse, Privat, 1981, p. 205-206.

(40) Si l'on sait quand une détention débute, on ignore le plus souvent quand elle se termine. La lettre de cachet ne comporte pas en effet de durée de détention, pratique qui ne se répand que dans la dernière décennie de l'Ancien Régime après la circulaire Breteuil visant à mieux contrôler la procédure des lettres de cachet (1784).

(41) On compte par exemple sept prisonniers dans le fort de l'Île Sainte-Marguerite en décembre 1789, cinq prisonniers à la Tour de Crest en janvier 1790.

(42) Estimation de Claude QuéTEL, De par le Roy, op. cit., p. 178. 
trière, dominent en 1789 le paysage des maisons de force par le nombre de prisonniers enfermés entre leurs murs. Le rayonnement de ces deux établissements, dû essentiellement à la faiblesse de la pension exigée pour les correctionnaires mais aussi à l'action du lieutenant général de police, dépasse les limites de la Capitale. Bicêtre est une véritable ville dans la ville : pas moins de 507 hommes y sont entrés « par différents ordres », majoritairement par ordre de police ou sentence judiciaire, au cours de l'année 1789. Un état général des prisonniers en date du 4 mars 1790 comprend les noms de 496 prisonniers mais on signale sur le document qu'ils étaient plus de 800 quelques mois auparavant ${ }^{43}$, soit une petite minorité des 3000 à 4000 " pensionnaires » du lieu à la fin de l'Ancien Régime, principalement des pauvres et des vénériens ${ }^{44}$. À la Salpêtrière, on compte le 29 août 1789101 femmes détenues par lettre de cachet. Elles ne sont plus que 77 en mars 1790. Les deux grands hôpitaux généraux parisiens se sont donc vidés rapidement de leurs détenus par ordre du roi dans les premiers mois des événements révolutionnaires. Dans le reste du royaume, outre quelques communautés religieuses importantes, par exemple la communauté de Maréville en Lorraine ${ }^{45}$ spécialisée dans la séquestration des aliénés où sont toujours recluses 66 personnes le 23 février $1790^{46}$, les correctionnaires sont éparpillés entre une multitude de couvents, refuges et monastères qui ne déclarent qu'un contingent fort limité de prisonniers retenus illégalement.

Conséquence du décret du 15 janvier 1790 pour le recensement des prisonniers " illégaux », consolidé par les articles 14, 15 et 16 du décret d'abolition, le fonds du Comité des lettres de cachet regorge d'états de détenus venant de tout le royaume. Ces documents sont souvent peu clairs et confus; 1'Assemblée ayant demandé expressément que figurent sur les états les noms des détenus par lettre de cachet ou «par ordre quelconque des agents du pouvoir exécutif », les religieuses du Bon Pasteur de Dijon signalent ainsi la présence parmi elles de 13 femmes dont l'une est entrée sur ordre de l'évêque, l'autre sur ordre du maire. Des jeunes filles mineures placées pour leur éducation par leurs parents sont aussi parfois incluses dans ces états et on a tôt fait de mêler les personnes élargies à celles encore renfermées, les condamnés en justice et

(43) $\mathrm{AN} \mathrm{D} / \mathrm{V} / 6$.

(44) Claude QuÉTEL, Les lettres de cachet, une légende noire, Paris, Perrin, 2011, p. 276-277.

(45) Maison dirigée par les Frères des Écoles Chrétiennes, aujourd'hui Centre psychothérapique de Nancy-Laxou.

(46) $\mathrm{AN} \mathrm{D} / \mathrm{V} / 1$. 
les correctionnaires, voire les vivants et les morts ${ }^{47}$. Malgré des erreurs, des omissions volontaires ou involontaires, les directeurs et supérieurs indiquent généralement soigneusement les renseignements requis. Les états se présentent sous la forme de tableaux inventoriant de manière plus ou moins exhaustive le nom du détenu, parfois son âge, la date des ordres, la date de son entrée dans la maison, voire de sa sortie, le motif de l'incarcération s'il est connu, et enfin une colonne « observations » et « commentaires » permet aux geôliers de donner leur avis sur l'éventualité d'une remise en liberté.

Le Comité a donc entamé un immense travail de recensement des milliers de prisonniers encore détenus début 1790. Il s'agit d'être en mesure d'évaluer quasiment au cas par cas le bien-fondé d'une libération et les bénéfices d'un maintien dans les fers. Les membres du Comité disposent d'autres éléments pour en juger : des centaines de lettres émanant de cette masse opaque, enchaînée mais avide de liberté, parviennent en effet à l'Assemblée nationale puis au Comité.

\section{Des victimes qui réclament justice : étude de leur discours}

Dès les premiers jours de l'été 1789 , les prisonniers du roi et prisonniers de famille supplient les députés de ne pas les abandonner à leur triste sort et de mettre fin au plus vite à leur détention. Leur discours s'articule autour de deux grands axes : d'abord l'exposé de leurs malheurs et la description de terribles conditions de vie, ensuite leurs revendications. Le gentilhomme Hermandfroy de Moréal, originaire de Dole, déploie une énergie à nulle autre pareille pour obtenir gain de cause. Sur l'avis du Comte de Dortan, député de la noblesse de Franche-Comté, il écrit plusieurs lettres au Comité, dès novembre 1789, depuis la sordide maison de Maréville en Lorraine :

« Depuis six ans et demi, je suis sous le poids d'une lettre de cachet qui n'est pas limitée, j'ai fait ici trois maladies mortelles, trois fois je me suis vu aux portes du tombeau, j'ai souffert des maux inimaginables et tous ceux qui sont inséparable [sic] d'un état de captivité ${ }^{48}$.

Et ce même discours dépeignant un traitement cruel et inhumain

(47) Le « tableau des personnes détenues en vertu de lettres de cachet et ordres du roi expédiés du bureau des Colonies » recense par exemple un certain Baron de Silly, originaire de Guadeloupe et mort en 1782 au château de Cardillac-sur-la-Garonne, AN D/V/4.

(48) $\mathrm{AN} \mathrm{D/V/1.}$ 
est repris dans tous les écrits des prisonniers. À Bicêtre, où l'heure est à la révolte et à la mutinerie au début de l'année 1790, Pierre Legent adresse un mémoire au comte de Castellane le 5 avril 1790; il s'exprime au nom de tous les prisonniers de l'hôpital :

« Nous sommes encore tous et au nombre de quatre cents, oprimés [sic] indignement par le plus cruel despotisme, au lieu d'adoucir nos maux, nos geôliers ou économes, hommes tout puissants ici, ont redoublé d'activité pour nous rendre plus malheureux, nous sommes mis dans les affreux souterrains où l'on est attaché par le cou à une chaîne grosse et courte, les fers aux pieds et aux mains $[\ldots] \gg^{49}$.

Et les « affreux souterrains » de Bicêtre font écho aux cachots de Maréville où le malheureux détenu voit « une multitude de rats monstrueux qui, du fond de la latrine, viennent pendant la nuit partager son grabat $\gg{ }^{50}$. Les mots sont puissants et le style éloquent, la vérité de la situation de prisonnier du roi souvent peu exagérée; de nombreuses familles au $\mathrm{XVIII}^{\mathrm{e}}$ siècle rechignent à payer la pension de leur parent détenu, le laissant dans une misère affligeante dont l'issue peut être fatale.

Le sombre tableau des conditions de détention s'accompagne d'un réquisitoire contre ceux qui en sont jugés responsables, au premier rang desquels figurent les ministres ou agents royaux qui ont délivré une lettre de cachet ou favorisé son expédition; la «tyrannie ministérielle » et le caractère véreux de ses agents constituent des arguments récurrents. Le roi est quant à lui le grand absent : certes, on ne remet pas en cause sa clairvoyance puisqu'on considère que les ministres et leurs agents sont seuls responsables de ces crimes, à l'insu du roi; pourtant on ne fait appel à lui que très rarement, au profit de la seule Assemblée nationale qui voit affluer des témoignages d'amour et de fidélité éternelle ${ }^{51}$. Mais les premiers accusés sont les demandeurs de la lettre de cachet, avant tout les membres de la famille de la victime qui l'ont calomniée en vue d'intérêts bassement matériels. Contre ses propres parents, on appelle à des poursuites et on demande réparation pour le préjudice incommensurable subi : perte de la liberté, santé délabrée, réputation et honneur salis, fortune spoliée. C'est en ce sens que plaide Hermandfroy de Moréal qui réclame

(49) $\mathrm{AN} \mathrm{D} / \mathrm{V} / 2$.

(50) $\mathrm{AN} \mathrm{D/V/1.}$

(51) Par exemple « Les souhaits d'un prisonnier de Bicêtre pour le Nouvel An, à messieurs les représentants de la Nation », s.d., AN D/V/2. 
d'abord le droit d'être jugé et ensuite celui d'attirer les foudres de la justice sur son accusateur :

« [...] je demande à être renvoyé par devant mes juges naturels, ce n'est pas dans une maison de force et sous le poids d'une lettre de cachet que je peux me défendre. Si à quelques égards je suis coupable, je puis aussi à bien d'autres me justifier, auparavant d'être condamné je dois être entendu, cela est de droit naturel [...]. Mais Monsieur, en réclamant contre moi la sévérité de la loy, je dois aussi implorer la justice contre l'homme pervers, contre le perfide ravisseur qui a abusé de ma jeunesse et de mon inexpérience [...], je demande donc qu'il me soit permis de me pourvoir contre cet homme criminel, contre tous ceux qui directement ou indirectement ont trempé dans cet indigne complot, contre tous ceux enfin qui aujourd'huy encore, abusent de ma triste situation pour me ravir l'héritage que m'ont laissé mes père et mère, en un mot Monsieur, je demande justice de différents attentats commis contre ma personne dans un état même d'innocence, attentats qui ne furent jamais revêtus d'aucune autorité légale $»^{52}$.

Libéré du fort de Brescou depuis six mois, le sieur Gonelle, de Lyon, se plaignant de l'état de dénuement extrême dans lequel il se trouve, prie les députés de l'Assemblée nationale en son nom et en celui de ses condisciples, « de leur assurer par un décret, au moins l'étroite subsistance qu'ils avoient en prison du consentement de leurs détenteurs ou d'obliger ceux-ci à les recevoir et alimenter chez eux $\|^{53}$. Le sieur Macdonagh, gentilhomme irlandais enfermé pendant plus de douze ans au fort des Îles Sainte-Marguerite à la sollicitation de sa hiérarchie militaire, souhaite obtenir remboursement de la pension versée pendant huit ans au commandant du fort, au motif que ce dernier l'a privé de nourriture pendant toutes ces années ${ }^{54}$.

Boniface de Castellane au cours de la séance du 13 mars 1790 avait exprimé le désir que le décret d'abolition accorde aux opprimés tous les recours qu'ils pourraient porter contre les auteurs de leur détention ${ }^{55}$, mention qui ne figure toutefois pas dans la version finale du décret ${ }^{56}$. Des

(52) AN D/V/1.

(53) $\mathrm{AN} \mathrm{D} / \mathrm{V} / 2$.

(54) AN D/V/6.

(55) Archives Parlementaires, t. XII, p. 161.

(56) L'article 11 rend cependant obligatoire la communication des pièces de la procédure par les ministres aux prisonniers. 
demandes en réparation d'actes arbitraires, d'abus de pouvoir et d'arrestations en vertu de lettres de cachet sont cependant bien présentées dans les années 1790-1792 devant les tribunaux à Paris ${ }^{57}$, cette démarche se trouvant fortement encouragée alors par certains journaux ${ }^{58}$ et clubs $^{59}$. Ces procès restent marginaux, mais sur des rapports de la Commission des lettres de cachet instituée en novembre 1791, quelques particuliers obtiennent en revanche de l'Assemblée le versement d'argent « à titre d'indemnité des frais de détention et de traduction arbitraire $»^{60}$. Une « Société des victimes du pouvoir arbitraire »s'est spécialement créée le 11 octobre 1790 dans le but d'obtenir dommages et intérêts pour les torts subis sous l'Ancien Régime. Elle compte dix-huit membres début 1791, principalement des militaires ou d'anciens agents du roi réclamant contre leur destitution et leur disgrâce, et un seul ex-détenu par lettre de cachet, le sieur Dagonneau de Bussy, de Dijon, détenu pendant 20 ans aux Îles Sainte-Marguerite, ce qui l'a privé de la succession de sa mère ${ }^{61}$.

De l'amorce de la Révolution jusqu'en 1791, les prisonniers par ordre du roi, mus par l'espoir d'une libération prochaine, voire d'une réparation, sont donc particulièrement actifs et entendent participer aux débats de l'Assemblée. Mais dans quelle mesure parviennent-ils réellement à émouvoir et à toucher ceux qui sont directement concernés par leur sort à savoir leur famille, leurs geôliers, les autorités locales et judiciaires concernées et enfin les députés de l'Assemblée nationale?

\section{Une perception ambivalente des prisonniers par lettre de cachet}

Les familles sont à l'origine de l'immense majorité des lettres de cachet qui en 1789 retiennent des hommes et des femmes en détention au nom de la sauvegarde de l'honneur et des intérêts familiaux, en réalité pour des motifs souvent légers et sans proportion avec la peine infligée, ou même imaginaires. Elles craignent certes d'être accusées de complicité et de participation à des pratiques arbitraires et despotiques, mais avant

(57) A. Dounarche, « Les victimes des lettres de cachet devant les tribunaux de la Révolution », Grande Revue, 15 juillet 1905, p. 20.

(58) Voir par exemple Le Moniteur du vendredi 23 avril 1790.

(59) Le Club des Droits de l'Homme invite dès le 27 avril 1790 tous les citoyens à lui faire connaître les actes d'oppression dont ils ont été victimes.

(60) Rapport de la Commission des lettres de cachet sur la pétition d'Antoine Buffel, chirurgien, traduit de La Canée en France, détenu au fort de Notre Dame de la Garde, en vertu d'ordres arbitraires, Paris, Imprimerie nationale, s.d., p. 7.

(61) $\mathrm{AN} \mathrm{D/V} / 4$. 
toutes choses le retour du banni au sein du foyer. En septembre 1790, le sieur Quittet présente une demande officielle au bailliage de Poitiers et au Comité des lettres de cachet afin que son frère Louis-Joseph, dément, reste détenu, expliquant « [qu'il a] voulu par cette démarche ne pas être rangé au nombre des gens qui ont eu recours aux ordres pour vexer leurs parents $\gg{ }^{62}$.

Les familles tentent d'empêcher la libération de leur parent en se tournant vers les autorités municipales ou judiciaires locales, suivant en cela l'article 6 du décret d'abolition des lettres de cachet, selon lequel les prisonniers seront élargis dans un délai de trois mois si aucune plainte n'est déposée contre eux devant les tribunaux. Le père de Jeanne-Baptiste de La Chapelle, recluse au Bon Pasteur de Dole, manifeste ainsi le souhait de paraître devant le maire de la ville pour lui présenter « actes, titres et papiers capables de justifier légalement les griefs relevans [...] contre cette fille $\rangle^{63}$. La famille de Claude-François Perrey se réunit de nouveau en assemblée de famille avec présentation de témoins, afin de rédiger un mémoire adressé à l'Assemblée nationale pour obtenir que le jeune homme âgé de 21 ans demeure jusqu'à ses 30 ans chez les Cordeliers de Seillières qui ne veulent plus le garder, demande appuyée par le lieutenant particulier du bailliage de Salins ${ }^{64}$. Le retour du mauvais sujet est susceptible de ramener le désordre dans la famille et dans le village; voilà un argument qui, en des temps de troubles et d'instabilité, est loin de laisser insensibles les autorités.

À l'instar des familles, beaucoup de directeurs et de supérieurs en charge de la garde des détenus du roi et des prisonniers de famille cherchent à éviter toute compromission. Le correctionnaire apparaît comme un poids et la charge de le surveiller et de l'accueillir comme une mission accomplie à contrecœur alors même que la pension payée pour sa subsistance représente pour les maisons religieuses une source de revenus appréciable. Mais compassion et pitié sont également des sentiments qui animent les geôliers. Le commandant du Fort Saint-André de Salins s'apitoie de la dureté avec laquelle le prêtre Valentin d'Ecuillon est traité :

« Je dois déclarer que depuis que j'ay été nommé au commandement de cette forteresse frontière, $j$ 'ay remarqué avec douleur que la famille de

(62) Ibid.

(63) Ibid.

(64) AN D/V/3. 
cet ecclésiastique l'a laissé dans sa prison couvert de haillons et ne lui a jamais fourni de bois pendant les froids les plus rigoureux qui existent sur cette montagne l'hiver. C'est un miracle qu'il n'ait pas péri. J'en ay informé Mrs de Breteuil et Villedeuil, successeurs au département de M. Bertin. Je les ay suplié d'exercer leur charité pour rétablir la santé dud. prêtre, de le faire transférer dans les prisons de l'officialité de Lyon, son diocèse, son juge et domicile naturel. Jamais de réponse de leur part $»^{65}$.

Certains députés se posent en défenseurs de correctionnaires enfermés dans leur circonscription et plaident en leur faveur auprès du Comité ou à l'Assemblée. Le soutien vient aussi de la communauté villageoise et des autorités locales : à Valfroicourt dans les Vosges, tous les notables composant la municipalité prennent fait et cause pour Richard Bastien, détenu depuis plus de deux ans à Maréville à la sollicitation de son frère, prêtre du village, et de son épouse, accusés d'entretenir une liaison scandaleuse $^{66}$. Plus étonnante encore est l'intervention de la religieuse Julienne Ruaut pour la libération du marquis de Bouilly de Resnon. Ayant été conduite dans l'appartement de ce dernier au cours d'un voyage au Mont-Saint-Michel « pour en voir les curiosités », elle s'émeut de sa situation et écrit au président de l'Assemblée nationale en juillet 1790 par 1'intermédiaire du suppléant des députés de la sénéchaussée de Rennes ${ }^{67}$. Les prisonniers dont nous gardons la trace grâce aux mémoires envoyés au Comité paraissent généralement fort bien informés des débats et des décrets adoptés par l'Assemblée, comme du rôle du Comité; ils ont vraisemblablement toujours bénéficié d'une aide extérieure, de la part d'un député, d'un parent, voire d'une connaissance. La grande majorité des détenus, privée de moyens de communication avec l'extérieur et ignorante des débats intenses au cœur desquels elle se trouve, ne connaît pas la même chance.

\section{Les conséquences du décret de mars 1790}

\section{Une application délicate et complexe}

Le Comité des lettres de cachet est plus sollicité que jamais par les familles et les correctionnaires après l'adoption du décret d'abolition, alors que son rôle se limite désormais à un simple contrôle de l'appli- 
cation du texte qui relève des seuls pouvoirs exécutifs et judiciaires sur place. À la lecture de l'article 16 du décret qui désigne explicitement les personnes chargées de son exécution dans le royaume, on comprend combien leur tâche s'annonce ardue :

«L'Assemblée nationale rend les commandants des prisons d'État, les supérieurs des maisons de force et maisons religieuses, et tous les détenteurs de prisonniers enfermés par ordre illégal, responsables, chacun en ce qui le touche, de l'exécution du présent décret, et elle charge spécialement les tribunaux de justice, les assemblées administratives de départements et de districts, et les municipalités, d'y tenir la main chacun en ce qui le concerne $»^{68}$.

Les intervenants sont nombreux et les responsabilités morcelées entre agents de l'État, juges et directeurs de maisons de force dans des dossiers où le mystère et l'opacité sont rois. Les geôliers, déjà placés au cœur du dispositif par le décret du 15 janvier, sont en première ligne. En juin 1790, désemparé, le Frère André, de la communauté de Maréville, écrit au Frère Bertier, directeur des Frères des Écoles chrétiennes de la rue Notre-Dame des Champs à Paris, pour le prier d'aller voir en personne le Comte de Castellane au sujet du cas Alexis Clerc :

" Comme Monsieur de Castellane est à n'en pas douter accablé d'ouvrage, il pourroit bien se faire qu'il tardât trop longtemps à nous honorer d'une réponse, c'est pourquoi [...] nous vous prions de lui rendre une visite au reçu de la présente et de le supplier de vous donner au moins de vive voix tous les éclaircissements nécessaires touchant la manière de mettre à exécution le décret dans tous ses articles $»^{69}$.

Le cas d'Alexis Clerc condamné par arrêt du parlement de Besançon à une peine de prison perpétuelle pour avoir frappé son prieur, pose question : le supérieur de Maréville juge qu'en vertu du décret, il doit être transféré à Besançon afin que son procès soit révisé, quand l'accusé soutient qu'il doit être libéré sur le champ en se basant sur une interprétation fine des articles 5 et $7^{70}$. Juges, supérieur, agents du pouvoir central, personne ne souhaite prendre une telle décision et tous se tournent vers le Comité afin d'obtenir une réponse. Trois mois après l'adoption du décret,

(68) Archives Parlementaires, t. XII, p. 203.

(69) AN D/V/2.

(70) Ibid. 
c'est donc l'anarchie la plus totale; entre l'hétérogénéité des prisonniers (condamnés en justice, fous frappés d'interdiction, prisonniers de famille par lettre de cachet ou par « ordre particulier» sur simple demande des familles...), la diversité des dispositions prévues par le décret du 16 mars 1790 et la confusion qui règne dans le partage des responsabilités pour son application, la situation paraît difficilement contrôlable dans un royaume en pleine effervescence révolutionnaire.

Le décret du 16 mars s'appuie en effet sur des institutions nouvelles et une organisation administrative à peine esquissée ${ }^{71}$. Le Comité des lettres de cachet n'hésitant pas à rappeler à l'ordre ou à récriminer les agents ou geôliers tardant à appliquer le décret, il reçoit des réponses de la même teneur que celle du président du directoire du district de Nancy qui décrit le 13 août 1790 les conditions difficiles dans lesquelles s'exerce sa mission :

« Le Directoire du district de Nancy n'étoit pas encore formé, lorsque vous luy avés mandé que le Comité des lettres de cachet avoit appris avec douleur que l'article 9 du décret de l'Assemblée nationale sanctionné le 26 mars dernier, confié à sa vigilance, n'avoit pas reçu d'exécution $»^{72}$.

Compte rendu est fait ensuite de l'application dans la maison de Maréville de l'article 9 du décret touchant les personnes prétendument démentes, application marquée par des querelles de pouvoir entre les différentes autorités locales. La municipalité de Nancy pensant « qu'elle devrait se charger de la surveillance prescritte aux directoires de district par l'article 9 du décret $»^{73}$ a nommé un commissaire pour la visite des fous, assisté du procureur de la commune et du procureur du roi au bailliage de Nancy. Les détenus sont interrogés par les juges et examinés par des médecins, des procès-verbaux des interrogatoires et des observations des médecins sont dressés; à la suite de quoi le bailliage de Nancy se prononce sur le sort de chacun des détenus.

En vertu des visites et des informations recueillies auprès des geôliers, les districts sont tenus d'adresser des états des détenus ou encore des certificats attestant qu'ils n'ont rien reçu de la part des maisons de force de leur district aux directoires des départements qui statuent sur le sort des correctionnaires et se chargent d'adresser les états au Comité des lettres de

(71) Les municipalités sont créées par décret du 14 décembre 1789, les départements par décret du 22 décembre suivant.

(72) $\mathrm{AN} \mathrm{D} / \mathrm{V} / 4$.

(73) Ibid. 
cachet. Dans les faits, les rôles respectifs des autorités locales et judiciaires paraissent mal définis et l'application de la loi plutôt brouillonne, malgré le zèle certain de l'administration et des juges. Même au sommet de l'État, les attributions des comités révolutionnaires s'entremêlent et se chevauchent dangereusement. Mirabeau semble en profiter en essayant de se dispenser de l'étude qu'on lui a confiée au profit du Comité de mendicité ${ }^{74}$, ce qui lui vaut un rappel à l'ordre de la part de Barère en novembre 1790 :

«Le Comité a reçu Monsieur, la lettre que vous avez bien voulu lui écrire au sujet de votre travail sur les maisons de correction. Le Comité de mendicité s'occupe de ce qui concerne les maisons de correction relativement à sa partie; mais il est une partie de ce travail qui appartient au Comité des lettres de cachet; c'est l'établissement et l'organisation des maisons de correction qui doivent recevoir ceux que les jugemens de famille condamneront à perdre leur liberté conformément à ce qui est porté par les décrets de l'organisation judiciaire $»^{75}$.

Le Comité de mendicité, en ce qui concerne les vagabonds et mendiants ${ }^{76}$, le Comité de législation pour les condamnés en justice et le Comité des lettres de cachet pour les « corrections » familiales, sont en effet tous trois impliqués dans la fondation d'un nouvel ordre carcéral. Quant au Comité ecclésiastique, il se voit également interpellé par quelques religieux détenus par ordre illégal ${ }^{77}$. Malgré ou à cause précisément de la précision du décret d'abolition des lettres de cachet, la jeune administration révolutionnaire est dépassée par la tâche qui lui incombe. Le décret est en outre critiqué et ses échecs souvent flagrants. Alors même que le texte s'attache à préparer l'élargissement et l'avenir des correctionnaires en leur assurant une subsistance destinée à les empêcher de sombrer dans de nouveaux égarements dus à la misère et à l'abandon (art. 12 et 13 du décret), beaucoup d'entre eux restent voués à la marginalité et à l'errance car rejetés par leur famille et par la société ${ }^{78}$.

(74) Voir Henri de BÉGOUEN, art. cit., p. 495-496.

(75) AN D/V/6.

(76) Au sujet de l'action du Comité de mendicité, voir Alexandre Tuetey, L'Assistance publique à Paris pendant la Révolution. Documents inédits, recueillis et publiés par A. Tuetey. volume I, Les hôpitaux et hospices, 1789-1791, Paris, Imprimerie nationale, 1895. Au début de ses travaux en avril 1790, le Comité envoie des Commissaires dans les hôpitaux et dépôts de Paris.

(77) $\mathrm{AN} \mathrm{D/V} / 6$.

(78) La Demoiselle Laudois se trouve par exemple toujours au couvent de la Charité de Guingamp en décembre 1790, car ses parents refusent de la reprendre dans leur foyer. AN D/V/6. 
Le texte pèche aussi par son approximation et sa subjectivité; l'article $1^{\mathrm{er}}$, au cœur du dispositif mis en place par le décret, témoigne des hésitations des députés à l'encontre de ces prisonniers de famille si nombreux, si mystérieux et si redoutés; tous doivent être remis en liberté, " à moins [...] que leurs père, mère, aïeul ou aïeule, ou autres parents réunis, n'aient sollicité et obtenu leur détention d'après des mémoires et demandes appuyées sur des faits très graves [...]». En ajoutant également aux exceptions les personnes prétendument frappées de folie, le décret rend de fait possible le maintien en détention de la grande majorité des prisonniers de famille, le plus souvent détenus sur présentation d'un mémoire rédigé en assemblée de famille, fréquemment accusés de folie ou du moins de « dérangements » et « dérèglements », et dont le sort est laissé à l'appréciation fort subjective des accusations portées contre eux. Car pour les familles, il est aisé d'évoquer des faits graves, sans aucune preuve à l'appui; l'ancienneté de certaines affaires et surtout l'opacité et le secret entourant la procédure des lettres de cachet rendent l'application du décret et le profond désir de justice qu'on peut y lire presque vains. D'autant que, par manque d'information et sans maitrise de la situation, les exécutants du décret s'empêtrent dans leurs contradictions. La Dame Fournier présente un mémoire contre son mari le 20 août 1790. Ce dernier a tenté de l'assassiner avant d'essayer de mettre fin à ses jours, une interdiction a été prononcée puis une lettre de cachet a été expédiée contre lui. Cette dame a de surcroît obtenu, après le décret du 16 mars, une sentence de la mairie de Louens pour la continuation de la détention de son époux chez les Frères des Écoles chrétiennes. Les faits sont ici très sérieux, constatés en justice; pourtant, après s'être informé sur ce cas, le Comité des lettres de cachet s'aperçoit que l'homme a simplement été remis en liberté le 23 juillet sans même que sa famille en soit avertie ${ }^{79}$.

Le premier article du décret d'abolition cristallise de nombreuses critiques; un particulier nommé Moreau de Maisonneuve, ancien magistrat aux colonies, fustige dans un mémoire au Comité les exceptions posées à la libération des prisonniers de famille, pour cause d'accusations portant sur des « faits très graves » :

« J'ose me permettre de croire qu'une pareille disposition va devenir l'origine et la source de nouvelles opressions [sic] d'autant plus désas-

(79) AN D/V/4. 
treuses qu'elles seront couvertes du manteau d'une loi infiniment respectable à tous égards $»^{80}$.

Et l'ancien magistrat de pointer les insuffisances du décret et les obstacles insurmontables entravant son application : à qui s'adresser pour connaître les motifs d'une détention placée sous le sceau du secret, comment obtenir communication des pièces de la procédure? Malheureusement les bureaux des ministres sont en effet bien incapables de retrouver la trace des prisonniers et peu songent à se tourner vers les archives des anciennes intendances où cependant se trouve conservé l'essentiel des dossiers ${ }^{81}$.

Globalement, De Maisonneuve dénonce « cet espère de respect que le décret paraît anoncer [sic] de la part de l'auguste Assemblée nationale pour les parens [sic] qui ont obtenu les détentions ». Ce respect de l'ordre établi, de l'autorité familiale et patriarcale conçue comme inséparable de l'autorité de l'État, reste profondément ancré dans l'action et les réflexions des députés autour de l'épineuse question des lettres de cachet. En finir avec l'oppression, certes, mais surtout préserver l'ordre social et l'ordre familial, tels sont en 1790 les principes directeurs des hommes de la Révolution.

\section{De la Commission des lettres de cachet aux tribunaux de famille : les révolutionnaires à la recherche d'un substitut aux lettres de cachet}

Les archives témoignent d'une activité intense du Comité des lettres de cachet tout au long de l'année 1790; au cours du premier semestre 1791 , il continue de veiller à l'exécution du décret dans les départements, en n'hésitant pas à rappeler à l'ordre des directeurs et supérieurs de maisons de force, comme des autorités locales accusées de ne pas avoir satisfait en particulier aux articles 9, 14 et 15 du décret, au premier rang desquels la municipalité de Paris ${ }^{82}$. À l'heure du bilan, en août 1791, le constat est plutôt sévère :

«Les opérations du Comité devroient être finies et le seroient en effet,

(80) $\mathrm{AN} \mathrm{D} / \mathrm{V} / 2$.

(81) Voir les archives souvent touffues, conservées dans les séries $C$ « Intendance » des Archives départementales.

(82) AN D/V/5. 
si les personnes chargées de l'exécution des décrets ci-devant cités [décrets du 15 janvier et du 16 mars 1790], y avoient obéi; mais plusieurs détempteurs de prisonniers, en vertu d'ordres arbitraires, bien loin d'avoir rempli les dispositions des articles XIV et XV du décret du mois de mars, qui leur prescrivoient de dresser et d'envoyer un état des prisonniers qui auroient été élargis, interrogés et visités, renvoyés par devant les tribunaux, ou qui garderoient encore prison, n'ont pas même envoyé à l'Assemblée nationale les états qu'elle avoit demandé par son décret du mois de janvier 1790. Au surplus dans plusieurs maisons, où sont détenues des personnes pour cause de démence, on n'a point fait la visite prescrite par l'article IX du décret du mois de mars 1790, en sorte qu'il se trouve des particuliers privés de leur liberté qui auroient dû être relâchés six semaines ou trois mois après la publication de ce décret. Nous en avons fait plusieurs fois des plaintes aux ministres qui en ont fait part à tous les départements $[\ldots] »^{83}$.

À l'automne 1791, le Comité des lettres de cachet disparaît dans le sillage de l'Assemblée constituante. Il est remplacé en novembre par une Commission des lettres de cachet, instituée par la Législative en vue d'examiner les archives de l'ancien Comité et les plaintes de victimes «d'ordres illégaux ${ }^{84}$. Elle ne se réunit que sept fois du 16 novembre 1791 au 8 septembre $1792^{85}$ avant de se séparer sur un projet de décret qui justifie sa dissolution, constatant « la cessation active depuis longtemps de toute espèce de réclamation contre des détentions illégales » et que « depuis [la] dernière loi sur la suppression totale des maisons religieuses et des congrégations, aucune maison de détention ne peut subsister que celles qui sont immédiatement sous la vigilance des corps administratifs $»^{86}$.

(83) AN D/V/6.

(84) Il est à noter que ces « ordres illégaux » ne sont pas uniquement des lettres de cachet, mais également toutes les sentences judiciaires rendues par les conseils du roi : voir par exemple le Rapport de la Commission des lettres de cachet sur la pétition du sieur Joseph Broqua, victime de trois arrêts du Conseil des Dépêches. S.l., s.d.

(85) AN D/V/7, registre de la Commission des lettres de cachet. Le président de la Commission est Charles Duval.

(86) Ibid. L'article 2 du quatrième projet de décret de la Commission stipule que « les conseils généraux des communes dans l'étendue desquelles il se trouve des maisons de détention qui n'auroient pas été visitées, aux termes de la loi du 26 mars 1790 et qui ne le seroient pas dans le mois de la publication du présent décret demeureroient personnellement responsables des dommages et intérêts que les détenus seront admis à répéter contre eux depuis l'époque de la publication du 26 mars $1790 »$. AN D/V/7. 
Le Comité puis la Commission des lettres de cachet ont entériné la condamnation sans appel de la détention arbitraire, en dehors du champ de la loi et de la justice, par le simple fait du Prince, que permettait l'institution des lettres de cachet. La nécessaire « correction » des mauvais sujets n'est pourtant pas remise en cause et on s'inquiète dès 1789 des conséquences de la disparition des lettres de $"$ petit cachet ${ }^{87}$ sur l'ordre familial et social. Les députés et les membres du Comité sont donc mus par le désir de trouver un substitut à la disparition de ces ordres conçus comme des garants de l'autorité dans la famille comme dans le royaume. C'est dans cette optique que Mirabeau s'est vu confier par le Comité la rédaction d'un rapport sur les " maisons d'amélioration ${ }^{88}$ destinées à recevoir les correctionnaires de l'Ancien Régime, fils de famille fauteurs de trouble et autres parents " dérangés ». C'est également pour répondre à ce besoin de prise en charge des problèmes familiaux, caractéristique du dernier siècle de l'Ancien Régime, que les Révolutionnaires créent les tribunaux de famille par décret du 16 août 1790 au sein d'un nouveau système judiciaire qui promeut l'arbitrage, les « bureaux de paix » et de « conciliation $»^{89}$.

Une tendance contradictoire s'affirme avec force dans le sillage du débat sur les lettres de cachet : d'une part, les législateurs révolutionnaires s'opposent à l'existence d'un pouvoir patriarcal et familial oppressif et cherchent à favoriser l'émancipation de l'individu; d'autre part, ils ne souhaitent pas laisser la famille réguler seule ses conflits, alors même que le recours à l'État est passé dans les mœurs et qu'une forte demande d'assistance perdure ${ }^{90}$.

À la fin de l'année 1792, il semble que, tant bien que mal, dans

(87) Appellation commune des lettres de cachet pour affaires de famille.

(88) Henri DE BÉGOUEN, art. cit., p. 491-512.

(89) Jacques Commaille, « Les formes de justice comme mode de régulation de la famille, questions sociologiques posées par les tribunaux de familles sous la Révolution française » dans Christian BIET, Irène THÉRY (dir.), La famille, la loi, l'État : de la Révolution au Code civil, Paris, Impr. Nationale, 1989, p. 274-289.

(90) André BurguiÈre, « Demande d'État et aspirations individualistes. Les attentes contradictoires des familles à la veille de la Révolution » dans Marie-Françoise LÉvy (dir.), L'enfant, la famille et la Révolution française, Paris, Olivier Orban, 1989, p. 25-32. 
un respect plus ou moins grand des articles du décret d'abolition, les maisons de force, châteaux forts, prisons d'État et maisons religieuses se soient vidés de la grande majorité de leurs prisonniers par lettre de cachet. L'étude des débats parlementaires comme du règlement au cas par cas d'un certain nombre de dossiers de détenus révèlent que la question des lettres de cachet a soulevé une réflexion beaucoup plus large sur la justice de l'Ancien Régime et le nouvel appareil judiciaire à bâtir dans le royaume. Les deux justices, celle, retenue, du roi et celle, déléguée, des juges s'enchevêtrent régulièrement dans les dossiers de lettres de cachet, la première venant pallier bien souvent les insuffisances de la seconde en la court-circuitant. À l'heure où il convient de statuer sur le sort des détenus sous le joug de la justice retenue du roi, le législateur cherche à entamer une démarche inverse, à savoir remettre dans la plupart des cas ceux qui restent encore des accusés entre les mains de leurs juges naturels, démarche cependant inachevée, les fondements du nouvel appareil judiciaire et administratif mis en place par la Révolution étant alors à peine jetés.

Le décret d'abolition et les conséquences concrètes de son application sur le sort des anciens correctionnaires et prisonniers du roi portent en germe les principes du nouveau système pénal centré sur le carcéral institué par la Révolution; ils ont aussi initié la réflexion des Révolutionnaires sur la régulation des conflits d'ordre privé et familial par l'État. Il n'est pas question en effet d'abandonner les familles à elles-mêmes quand l'État est devenu l'arbitre et l'autorité régulatrice incontournable des tensions les déchirant. L'abolition d'une institution symbolique de la monarchie absolue et de son gouvernement, et avec elle, la destinée de milliers d'anonymes érigés en martyrs avant de subir le poids du soupçon et de la peur, ont donc sans conteste contribué à engager et orienter les débats autour de la fondation d'un ordre judiciaire et social nouveau qui ne renie cependant pas entièrement l'héritage de l'Ancien Régime.

Jeanne-Marie JANDEAUX EHESS-CRH (Doctorante à l'EHESS Archiviste-paléographe Conservateur des bibliothèques à l'Université de Nice SophiaAntipolis) jm.jandeaux@gmail.com 